\title{
Effects of two-step post heat-treatment in palm oil on the properties of oil palm trunk particleboard
}

\begin{abstract}
Chemical, thermal properties, surface characteristics, termite resistance and physicomechanical properties of urea formaldehyde-bonded oil palm trunk particleboard post heattreated in palm oil were investigated. Oven dried samples were immersed in palm oil for $24 \mathrm{~h}$ prior to expose to temperature of 180,200 and $220^{\circ} \mathrm{C}$ in a laboratory oven for $2 \mathrm{~h}$. Alternation in the chemical composition of treated samples was investigated using Fourier transform infrared spectroscopy (FTIR) analysis. Thermal properties analysis was conducted using Thermogravimetric (TG) and Different scanning calorimetric (DSC) analysis. Surface characteristics were checked using scanning electron microscopy (SEM) while resistance against subterranean termite, Coptotermes curvignathus, was evaluated. Physcial and mechanical properties of the samples was assessed based on anti-swelling efficiency (ASE), water repellency efficiency (WRE), bending and internal bonding strength. Degradation of hemicellulose shown in FTIR spectra has contributed to the improvement in dimensional stability. Oil-covered particles as shown in SEM give the particleboard lower water uptake. Treated samples also possess better thermal stability and better resistance against termites as the weight loss caused by termites reduced from $25.6 \%$ to $11.0 \%$. Generally, improvement in dimensional stability accompanied by severe reduction in mechanical strength were recorded in the treated samples.
\end{abstract}

Keyword: Oil palm trunk; Oil heat treatment; Dimensional stability; Termite resistance; Thermal properties 